\title{
Vacuum Microwave-Assisted Aqueous Extraction of Polyphenolic Compounds from Avocado (Persea Americana) Solid Waste
}

\author{
Prodromos Skenderidis ${ }^{1, *(D)}$, Stefanos Leontopoulos ${ }^{1}$, Konstantinos Petrotos ${ }^{1}$ and Ioannis Giavasis ${ }^{2}$ \\ 1 Laboratory of Food and Biosystems Engineering, Department of Agrotechnology, University of Thessaly, \\ 41110 Larissa, Greece; sleontopoulos@uth.gr (S.L.); petrotos@uth.gr (K.P.) \\ 2 Laboratory of Food Microbiology and Biotechnology, Department of Food Science and Nutrition, \\ University of Thessaly, 43100 Karditsa, Greece; igiavasis@uth.gr \\ * Correspondence: pskenderidis@uth.gr; Tel.: +30-697-331-3565
}

check for updates

Citation: Skenderidis, P.; Leontopoulos, S.; Petrotos, K.; Giavasis, I. Vacuum

Microwave-Assisted Aqueous

Extraction of Polyphenolic

Compounds from Avocado (Persea

Americana) Solid Waste. Sustainability

2021, 13, 2166. https://doi.org/

$10.3390 /$ su13042166

Academic Editor: Antonia Terpou

Received: 18 January 2021

Accepted: 15 February 2021

Published: 18 February 2021

Publisher's Note: MDPI stays neutral with regard to jurisdictional claims in published maps and institutional affiliations.

Copyright: (C) 2021 by the authors. Licensee MDPI, Basel, Switzerland. This article is an open access article distributed under the terms and conditions of the Creative Commons Attribution (CC BY) license (https:/ / creativecommons.org/licenses/by/ $4.0 /)$.

\begin{abstract}
The extraction efficacy of avocado fruit peels (AP) and seeds (AS) with the use of vacuum microwave-assisted aqueous extraction (VMAAE) was optimized in this study, with regard to extract's total phenolic content (TPC), maximum antioxidant activity and minimal operational cost. Temperature (79.64 and $78.11^{\circ} \mathrm{C}$ for AP and 43.90 and $45.26^{\circ} \mathrm{C}$ for AS), time (11.89 and $11.75 \mathrm{~min}$ for $\mathrm{AP}, 10.18$ and $10.28 \mathrm{~min}$ for $\mathrm{AS})$, ratio of water to raw material (16.45\% and $10.02 \%$ for $\mathrm{AP}, 38.73 \%$ and $37.65 \%$ for AS) and microwave power (5708.04 and 5699.10 W for AP, 5549.08 and 4797.29 W for AS) were estimated statistically as the optimal conditions in order to achieve high rates of extracts with high TPC and antioxidant activity using the 2,2-diphenyl-1-picrylhydrazyl (DPPH $\left.{ }^{\bullet}\right)$ scavenging radical methods, respectively. VMAAE performed under these conditions resulted in received extracts with TPC (0.352 gallic acid equivalent-GAE/g fresh $\mathrm{AP} / \mathrm{min}$ and $0.124 \mathrm{GAE} / \mathrm{g}$ fresh AS/min). Furthermore, it was calculated the $\mathrm{DPPH}^{\bullet}$ radical scavenging activity was equal to $100 \mathrm{mg} / \mathrm{L}$ expressed in L of $0.104 \mathrm{~L} / \mathrm{min}$ for AP and $0.045 \mathrm{~L} / \mathrm{min}$ for AS. The results of our study may give a promising solution to avocado processing companies for further utilization of their waste.
\end{abstract}

Keywords: avocado by-products valorization; antioxidants; modeling of vacuum microwave extraction; response surface methodology; polyphenolic compounds

\section{Introduction}

The avocado plant (Persea americana Mill.) is grown worldwide in the tropics. Avocado fruits were consumed in Central America over the past 10,000 years [1]. Due to its high nutritional content, avocado fruit has recently attracted growing global interest [2]. Commercial varieties are mainly based on the hybrid varieties of Guatemala and Guatemalan-Mexican varieties [3].

Consumption of avocados as fresh fruit is widely used nowadays. Furthermore, its pulp is used by food, pharmaceuticals and cosmetic industries due to its high concentration in vitamins, proteins, polyphenols, fibers, minerals, unsaturated fatty acids and other beneficial phytochemicals [4]. During fruit processing the peels (AP) and seeds (AS) end up as by-products comprising of 30\% of total fruit weight [5,6]. Pro-anthocyanidins (procyanidin type A and B), catechins, quercetin, glycerides, triamcinolone acetaminophen, saponins, steroids, caffeoalkinic acid and coumaric acid are the main complex mixture of polyphenolic compounds found in avocado residues [7-12].

Several patented uses of avocado in cosmetics, food and medical field are currently reported in a review study. These patents indicates the application of avocado pulp and avocado by-products to produce foods like antioxidant tea, nutraceuticals creams, and natural extracts with antioxidant and anti-inflammatory activity [13]. Furthermore the effective use of a spray drier powder from avocado process waste water as a natural antioxidant in pork sausage production was recently presented [14]. 
The extraction techniques used in industrial food processing results in the isolation of the phytochemicals found in by-products that can be applied as active compounds in functional food, colorants, flavors, antioxidants, antimicrobial, and stabilizers [15]. In addition, the use of these wastes could contribute to the reduction of the environmental impact and costs in the food industry [16].

Regarding isolation and extraction of bioactive components from food residues many methods have been proposed [17-19]. Microwave-assisted extraction is characterized by faster extraction times smaller size of the required equipment, higher efficiency, reduced energy consumption (up to 85 times), lower amount of waste and exposure of bioactive components to thermal destruction $[20,21]$.

Furthermore, vacuum microwave-assisted aqueous extraction (VMAAE) has been reported as an innovative extraction technology and is widely recognized as "pure green" technology because of the absence use of organic solvents [22,23]. VMAAE can enhance the mass transfer process by promoting the diffusion of the active substances contained in extracted materials into the solvent using an intake pressure technique [24]. The thermal degradation and oxidation risks can be reduced by the vacuum as this can reduce the solvent's related boiling temperature. A comparison between VMAAE and the application of a standard microwave-assisted extraction (MAE) on extraction ability of vitamin C from several fruits and vegetables such as guava fruit, green pepper, soybean and tea leaves has demonstrated an increase of vitamin C yields of 53\%, 145\%, 20\% and 60\% respectively [25].

The aim of this research study was to demonstrate and model using a response surface methodology the efficacy of industrial-scale VMAAE of avocado peels and seeds regarding the yield of the total content of phenols, evaluating also the antioxidant activity of these extracts. Furthermore, relations of the extraction rate of the selected responses will be used in order to evaluate the operational cost using a Box and Behnken experimental design of 29 experiments performed in triplicate. The appropriate equations were developed in order to model and achieve the prediction of the total phenolic content (TPC) and the extract's antioxidant activity.

\section{Materials and Methods}

\subsection{Avocado By-Products}

Green skin avocado fruits of the "Pinkerton" variety collected in the year of 2019, were purchased from Crete Island in southern Greece. The peels (AP) and the seeds (AS) of the fruit were then separated and stored at $-28{ }^{\circ} \mathrm{C}$ until further mechanical shivering occurred using a sphere mill in order to powder the samples. The final AP and AS powders were sealed in plastic bags, weighing $2 \mathrm{~kg}$, and kept frozen at $-28{ }^{\circ} \mathrm{C}$ until extraction procedure.

\subsection{Chemicals}

Sigma Aldrich were supplied this research study with the appropriate reagents and chemicals such as Folin Ciocalteu 2N; 2,2-diphenyl-1-picrylhydrazyl (DPPH ${ }^{\bullet}$ ) (PubChem CID:74358); methanol (PubChem CID:887); anhydrous crystal-formed sodium carbonate (PubChem CID:10340); and gallic acid (PubChem CID:370) in order to complete it.

\subsection{Extraction Methodology}

Different quantities of water $(20,50$ or $80 \mathrm{~L})$ depending on the dilution ratio $(10 \%, 25 \%$ or $40 \%$ ) were used in each of the selected experimental conditions in order to extract $2 \mathrm{~kg}$ of each sample (AP or AS). Using a MAC-75 (Milestone Technologies Inc., Sorisole, Italy) multimode industrial microwave-type reactor for scale-up vacuum extraction, apparatus constructed by an Italian company (Milestone Technologies Inc., Sorisole, Italy) was used for the extraction experiments completed in Pellas Nature P. Co. (Edessa, Greece) in the year of 2020. The Box and Behnken statistical design (BBD) method which is a spherical design procedure was used in order to achieve the appropriate experimental plan. BBD is a widely used method in the optimization of extraction processes and it is used to select the experimental measuring point's network $[26,27]$. Four independent variables (A: extraction 
temperature: $40-60-80^{\circ} \mathrm{C}$, B: extraction time: $10-50-90 \mathrm{~min}, \mathrm{C}$ : microwave power: 2000 $4000-6000 \mathrm{~W} / \mathrm{cm}^{2}$ and D: Water solvent ratio to dry avocado tissue: $10-25-40 \%$ ) and two optimization objectives or "responses" ((a) maximization of total polyphenols and (b) Minimization of $\mathrm{IC}_{50}$ ) were taken into account. Optimization conditions were achieved by using the Design Expert v12 software, which calculated the optimal conditions and created the relevant diagrams and mathematical equations of surface response, a methodology that has been already tested successfully [28]. The pressure of the extractor was adjusted at $355 \mathrm{mBar}$ in all experiments.

The obtained extracts were filtered through porous Whatman paper grade 591 and were stored in a freezer at $-28{ }^{\circ} \mathrm{C}$ until further analysis. Centrifugation at $12,000 \mathrm{rpm}$ for 10 min was carried out for each sample before further analysis occurred. The obtained supernatant solution was used for the response's analysis.

\subsection{Determination of Total Phenolic Content (TPC) of the Extracts}

A previously published methodology was applied in this study [29]. Briefly, with $20 \mu \mathrm{L}$ of each extract, $1.58 \mathrm{~mL}$ of distilled water and $100 \mu \mathrm{L}$ of Folin-Ciocalteu reagent $(0.2 \mathrm{~N})$ were mixed. Afterwards, $300 \mu \mathrm{L}$ of $\mathrm{Na}_{2} \mathrm{CO}_{3}$ solution $(200 \mathrm{~g} / \mathrm{L})$ was added and samples were placed for $30 \mathrm{~min}$ in incubation at $43^{\circ} \mathrm{C}$ in the dark. The absorbance was then measured at $765 \mathrm{~nm}$. For the calculation of the TPC contained in samples, the calibration curve of gallic acid expressed as gallic acid equivalent (GAE) in $\mathrm{mg} / \mathrm{g}$ of the fresh byproduct was used and $50-500 \mathrm{mg} / \mathrm{L}$ was determined as the linearity range of standard gallic acid. TPC yield was expressed as $\mathrm{mg}$ of gallic acid equivalent per $\mathrm{g}$ of fresh weight (fw) of avocado by-product.

\subsection{Total Antioxidant Capacity of the Extracts (2,2-Diphenyl-1-Picrylhydrazyl (DPPH•) Method)}

A methodology presented by Brand-Williams et al. [30], was applied in this study. Different concentrations of distilled water were used in order to dissolve the samples that used also in the method of TCP. Afterwards, the products from the avocado samples were then mixed with $1 \mathrm{~mL}$ of DPPH radical $(100 \mu \mathrm{M})$ methanol solution. Due to several advantages, DPPH radical assay is the most appropriate and thus it has been chosen among other methods used for the estimation of the antioxidant activity of avocado extracts. In order to achieve the appropriate reaction, the contents were vigorously mixed and incubated at room temperature in the dark for $20 \mathrm{~min}$. The sample was then centrifuged for $10 \mathrm{~min}$ at 10,000 rpm and the final absorbance was read at $517 \mathrm{~nm}$. DPPH methanol was used as control while methanol solutions of the tested extracts were used as blank. In order to minimize statistical error, the experiments were repeated three times. Equation (1) was used in order to determine by calculations the percentage of the radical-scavenging capacity (RSC) of the extracts.

$$
\% \mathrm{DPPH}^{\bullet} \text { radical scavenging activity }=((\text { Abs control }- \text { Abs sample }) / \text { Abscontrol }) \times 100
$$

where: Abs sample and Abs are the absorbance values of the tested sample and the control. In order to measure the radical-scavenging capability of the extracts, the value of the $\left(\mathrm{IC}_{50}\right)$ was calculated using the graph of the \% RSC versus the extracts concentration content. The $\mathrm{IC}_{50}$ estimates the concentration of the extract caused by $50 \%$ scavenging of $\mathrm{DPPH}^{\bullet}$ radical and the lower extracts concentrations needed, the higher antioxidant activity is the sample. Finally expressed as the Extract Equivalent Volume (EEqV) $\mathrm{IC}_{50}$ of $\mathrm{DPPH}^{\bullet}$ radical scavenging activity equal to $100 \mathrm{mg} / \mathrm{L}$ expressed in L (Equation (2)):

$$
\mathrm{EEqV} \mathrm{R} \mathrm{IC}_{50} \mathrm{DPPH}^{\bullet}=\left(\frac{V t \times 100}{\mathrm{IC}_{50} \mathrm{DPPHt}}\right) / \mathrm{W}
$$

where $W$ is the extracted avocado's by-product weight that was constantly equal to $2(\mathrm{~kg})$, $V t$ and $\mathrm{IC}_{50}$ of DPPHt are the whole volume in liters (L) of the extract and the half maximum inhibitory concentration at a given extraction time respectively. 


\subsection{Mathematical Modeling of Box-Behnken Designs}

An experimental Box-Behnken Design (BBD) methodology was applied in order to choose the testing points. This statistical approach is commonly used due to its proven effectiveness in improving extractions $[26,28,31]$.

For the determination of the BBD model four independent variables were chosen. These independent variables were: the extraction temperature $\left(\mathrm{A}=40-60-80{ }^{\circ} \mathrm{C}\right)$, the extraction time ( $B=10-50-90 \mathrm{~min})$, the microwave power $(\mathrm{C}=2000-4000-6000 \mathrm{~W})$ and the ratio of water solvent to $\mathrm{PP}(\mathrm{D}=10-25-40 \mathrm{~L} / \mathrm{kg}$ ) (Table 1$)$. TPC and antioxidant activity based on the $\mathrm{IC}_{50}$ of free radical scavenging $\left(\mathrm{DPPH}^{\bullet}\right)$ were chosen as responses due to their dependence on the extraction method $[32,33]$. The response variables were selected and adapted to a second order polynomial model equation obtained by response surface methodology (RSM) (Equation (3)):

$$
Y=\beta_{0}+\sum_{i=1}^{3} \beta_{i} X_{i}+\sum^{3}{ }_{i=1} \beta_{i i} X_{i i}^{2}+\sum_{i=1}^{2} \sum^{3}{ }_{j=i+1} \beta_{i i} X_{i} X_{j}
$$

Table 1. Extraction parameters coded levels-groups of the independent extraction' variables.

\begin{tabular}{ccccc}
\hline \multirow{2}{*}{ Independent Extraction' Variables } & \multirow{2}{*}{ Code Units } & \multicolumn{3}{c}{ Coded Variable Level } \\
\cline { 3 - 5 } & & $-\mathbf{1}$ & $\mathbf{0}$ & $\mathbf{1}$ \\
\hline Temperature & $\mathrm{A}$ & $40{ }^{\circ} \mathrm{C}$ & $60{ }^{\circ} \mathrm{C}$ & $80{ }^{\circ} \mathrm{C}$ \\
Time & $\mathrm{B}$ & $10 \mathrm{~min}$ & $50 \mathrm{~min}$ & $90 \mathrm{~min}$ \\
Power & $\mathrm{C}$ & $2000 \mathrm{~W}$ & $4000 \mathrm{~W}$ & $6000 \mathrm{~W}$ \\
Ratio of water to avocado peels (AP) or & $\mathrm{D}$ & $10 \%$ & $25 \%$ & $40 \%$ \\
seeds (AS) & & & & \\
\hline
\end{tabular}

The TCP and the $\mathrm{IC}_{50}$ of the $\mathrm{DPPH}^{\bullet}$ were the response variables $(\mathrm{Y}) . \mathrm{X}_{\mathrm{i}}$ and $\mathrm{X}_{\mathrm{j}}$ were the independent variables that controlling the responses and the model's regression coefficients were $\beta_{0}, \beta_{\mathrm{i}}, \beta_{\mathrm{ii}}$ and $\beta_{\mathrm{ij}}$ (intercept, linear, quadratic and interaction).

Design-Expert v12 (Stat-Ease, Inc., Minneapolis, MN, USA) was used as a mathematical program in order to select the final 29 set-point combinations. The determination of these points was applied in triplicate. For the maximization of the effect of the unexplained variability in the observed responses, experiments were randomized and each variable was classified as represented in Table 1 into three groups: -1 (low), 0 (intermediate) and +1 (high).

\subsection{Data and Statistical Analysis}

Regarding the cost evaluation of the extraction process, the TPC extraction rate was calculated. Equations presented in a previews study [28] was considered in order to estimate the rate of TPC (RTPC) obtained from AP and AS, considering also parameters such as the set-up time of the apparatus, the time used for the extraction and the volume of the mixtures extracted. These parameters are presented in Equation (4).

$$
\operatorname{RTPC}(\mathrm{mg} \mathrm{GAE} / \mathrm{g} \mathrm{fw}) / \min =\frac{C t V t}{t+K}
$$

$C t$ and $V t$ are respectively the TPC concentration of equilibrium in the liquid extract at a given extraction time $t(\mathrm{mg} / \mathrm{L})$ and the rest of the volume in liters (L) of liquid extract at a particular extraction time. $t$ and $K$ are the extraction time in min and the constant setup time of the apparatus, which was calculated to $15 \mathrm{~min}$.

Likewise, based on the Equation (5), the $\mathrm{EEqV}$ rate of $\mathrm{IC}_{50}$ of the $\mathrm{DPPH}^{\bullet}$ radical scavenging $\left(\mathrm{REEqVR} \mathrm{IC}_{50} \mathrm{DPPH}^{\bullet}\right)$ was measured.

$$
\mathrm{REEqVR} \mathrm{IC}_{50} \mathrm{DPPH}^{\bullet}=\frac{\mathrm{EEqV} \text { of } \mathrm{IC}_{50} \mathrm{DPPH}^{\bullet}}{t+K}
$$


Design-Expert v12 software was used for analysis of variance (ANOVA). Multiple regression analysis was completed using the surface response method. For each extracted avocado by-product, the ANOVA derived two respective surface response equations, correlating the performance and bioactive parameters measured with the four independent extraction parameters. Optimal values of $\mathrm{TCP}$ and $\mathrm{IC}_{50}$ of $\mathrm{DPPH}^{\bullet}$ radical scavenging for the extracts obtained was also estimated using the same program. Furthermore, MiniTab ${ }^{\circledR}$ 17.1.0 statistical software (Minitab LCC, State College, PA, USA) used for Pearson correlation test. The statistical significance was set at $p \leq 0.05$.

\section{Results and Discussion}

\subsection{Predicted Models of the Responses by Response Surface Methodology (RSM)}

Table 2 represents the results of the 29 BBD experiments linked to the extracts' TPC and the radical scavenging activity of the $\mathrm{DPPH}^{\bullet}$. By applying multiple regression analysis to the experimental data using the Design Expert software package v12, the quadratic equations linked the responses and the independent variables were developed and were evaluated by ANOVA Tables 3 and 4 . The $p$-value was used in order to conduct the verification of the significance of each coefficient. A greater corresponding coefficient was observed on smaller $p$-values [34].

The experimental results shown that the higher achieved TPC in extracts was 10.104 and $5.604 \mathrm{mg} \mathrm{GAE} / \mathrm{g}$ fw from AP and AS, respectively. Based on this result it can be assumed that the polyphenolic content of the peels was significant higher from the seed's TPC. These results agreed with the results presented in a research study completed by Calderón-Oliver et al. [35] as for the TPC of AS extracts (5.7 mg GAE/g) but they presented higher TPC in AP extracts (19.7 mg GAE/g). This difference was probably attributed to the moisture difference of AP by-products due to the drying pretreatment of AP that they followed in their method.

Between the TPC of the extracts and the measured $\mathrm{IC}_{50}$ of the DPPH radical scavenging of the extracts there was a negative Pearson correlation of -0.7 and -0.71 of AP and AS, respectively (results not shown). These results indicate that the antioxidant activity (the lower the $\mathrm{IC}_{50}$, the higher antioxidant activity) of the extracts is in accordance with the TPC as well as with the phenols profile as mentioned in a study completed by Kosinska et al. [8] who reported also that AP presented higher antioxidant activity than AS due to higher TPC. Data in Tables 3 and 4 indicate that the equations obtained of the predicted models shown in Table 5 are significant $(p<0.01)$ and with high predictive accuracy, as is concluded from the high $R^{2}$ values. The determination coefficient $\left(R^{2}\right)$ indicated the sum of the variations that the model interprets. The determined high values of the adjusted coefficient $\left(R^{2}\right.$ adj) have indicated that the models were significant. 


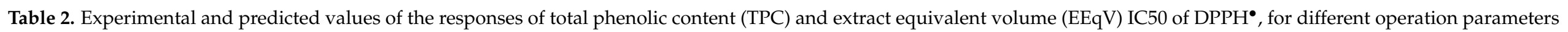
combinations based on the experimental design.

\begin{tabular}{|c|c|c|c|c|c|c|c|c|c|c|c|c|}
\hline \multirow{3}{*}{ Design Point } & \multirow{2}{*}{\multicolumn{4}{|c|}{ Independent Variables in Coded Values }} & \multicolumn{4}{|c|}{ Avocado Peel Responses } & \multicolumn{4}{|c|}{ Avocado Seed Responses } \\
\hline & & & & & \multicolumn{2}{|c|}{ TPC (mgGAE/g fw) } & \multicolumn{2}{|c|}{$\mathrm{EEqV} \mathrm{R} \mathrm{IC}_{50} \mathrm{DPPH}^{\bullet}(\mathrm{L})$} & \multicolumn{2}{|c|}{ TPC (mgGAE/g fw) } & \multicolumn{2}{|c|}{$\mathrm{EEqV} \mathrm{R} \mathrm{IC}_{50} \mathrm{DPPH}^{\bullet}(\mathrm{L})$} \\
\hline & A & B & $\mathrm{C}$ & $\mathbf{D}$ & Measured & Predicted & Measured & Predicted & Measured & Predicted & Measured & Predicted \\
\hline 1 & -1 & 0 & 1 & 0 & $6.573 \pm 0.06$ & 6.698 & $1.613 \pm 0.01$ & 1.516 & $4.354 \pm 0.06$ & 4.354 & $1.040 \pm 0.01$ & 0.932 \\
\hline 2 & 1 & 0 & 0 & 1 & $3.283 \pm 0.09$ & 3.283 & $0.584 \pm 0.00$ & 0.584 & $1.383 \pm 0.01$ & 1.383 & $2.775 \pm 0.04$ & 2.730 \\
\hline 3 & 0 & 0 & 0 & 0 & $6.602 \pm 0.04$ & 6.835 & $1.163 \pm 0.01$ & 1.555 & $2.021 \pm 0.03$ & 2.517 & $1.720 \pm 0.03$ & 1.634 \\
\hline 4 & 0 & 0 & -1 & 1 & $6.817 \pm 0.03$ & 6.820 & $0.871 \pm 0.00$ & 0.871 & $1.450 \pm 0.02$ & 1.422 & $2.750 \pm 0.04$ & 2.750 \\
\hline 5 & 0 & 0 & 0 & 0 & $6.354 \pm 0.01$ & 6.835 & $1.786 \pm 0.02$ & 1.555 & $2.688 \pm 0.04$ & 2.517 & $1.800 \pm 0.02$ & 1.634 \\
\hline 6 & -1 & 0 & 0 & 1 & $7.867 \pm 0.02$ & 7.867 & $2.516 \pm 0.01$ & 2.516 & $2.433 \pm 0.08$ & 2.433 & $1.750 \pm 0.03$ & 1.985 \\
\hline 7 & 0 & 0 & 0 & 0 & $7.052 \pm 0.04$ & 6.835 & $1.923 \pm 0.02$ & 1.555 & $2.646 \pm 0.08$ & 2.517 & $0.500 \pm 0.02$ & 1.634 \\
\hline 8 & 0 & 0 & 1 & 1 & $9.617 \pm 0.06$ & 9.292 & $2.759 \pm 0.03$ & 2.759 & $2.250 \pm 0.01$ & 2.278 & $1.575 \pm 0.02$ & 1.575 \\
\hline 9 & 0 & 1 & 0 & 1 & $7.300 \pm 0.04$ & 7.389 & $2.500 \pm 0.01$ & 2.531 & $5.217 \pm 0.04$ & 5.270 & $0.688 \pm 0.02$ & 0.443 \\
\hline 10 & -1 & 1 & 0 & 0 & $6.365 \pm 0.02$ & 6.204 & $1.282 \pm 0.04$ & 1.473 & $3.865 \pm 0.03$ & 3.939 & $0.872 \pm 0.02$ & 0.881 \\
\hline 11 & 1 & 0 & 1 & 0 & $10.104 \pm 0.08$ & 10.229 & $1.515 \pm 0.02$ & 1.696 & $2.927 \pm 0.01$ & 2.927 & $1.200 \pm 0.05$ & 1.254 \\
\hline 12 & 0 & -1 & 0 & -1 & $4.833 \pm 0.02$ & 5.065 & $0.667 \pm 0.01$ & 0.635 & $1.979 \pm 0.01$ & 1.925 & $3.500 \pm 0.07$ & 2.948 \\
\hline 13 & -1 & 0 & -1 & 0 & $7.594 \pm 0.01$ & 7.789 & $1.429 \pm 0.04$ & 1.345 & $1.906 \pm 0.02$ & 1.906 & $3.120 \pm 0.08$ & 3.132 \\
\hline 14 & 0 & 0 & 0 & 0 & $7.469 \pm 0.03$ & 6.835 & $1.256 \pm 0.06$ & 1.555 & $2.344 \pm 0.04$ & 2.517 & $2.000 \pm 0.06$ & 1.634 \\
\hline 15 & 1 & -1 & 0 & 0 & $7.458 \pm 0.05$ & 7.298 & $0.633 \pm 0.01$ & 0.598 & $1.479 \pm 0.01$ & 1.405 & $2.400 \pm 0.05$ & 2.599 \\
\hline 18 & 0 & 1 & -1 & 0 & $7.344 \pm 0.02$ & 7.292 & $1.786 \pm 0.04$ & 1.795 & $4.938 \pm 0.06$ & 4.809 & $0.880 \pm 0.03$ & 0.669 \\
\hline 19 & 1 & 1 & 0 & 0 & $7.281 \pm 0.02$ & 7.121 & $1.799 \pm 0.03$ & 1.653 & $5.604 \pm 0.08$ & 5.679 & $0.800 \pm 0.02$ & 0.463 \\
\hline 20 & -1 & -1 & 0 & 0 & $2.573 \pm 0.01$ & 2.413 & $0.427 \pm 0.02$ & 0.418 & $1.573 \pm 0.04$ & 1.498 & $3.400 \pm 0.04$ & 3.017 \\
\hline 21 & 0 & 1 & 1 & 0 & $8.500 \pm 0.06$ & 8.695 & $2.083 \pm 0.05$ & 1.966 & $5.833 \pm 0.03$ & 5.705 & $0.720 \pm 0.02$ & 0.629 \\
\hline 22 & 1 & 0 & 0 & -1 & $7.250 \pm 0.04$ & 7.250 & $4.348 \pm 0.06$ & 4.348 & $5.588 \pm 0.06$ & 5.588 & $0.500 \pm 0.01$ & 0.455 \\
\hline 23 & 1 & 0 & -1 & 0 & $6.135 \pm 0.02$ & 6.331 & $1.524 \pm 0.02$ & 1.525 & $4.438 \pm 0.04$ & 4.438 & $1.800 \pm 0.05$ & 1.974 \\
\hline 24 & 0 & -1 & 0 & 1 & $2.467 \pm 0.03$ & 2.699 & $0.563 \pm 0.01$ & 0.532 & $2.183 \pm 0.07$ & 2.130 & $2.525 \pm 0.04$ & 2.579 \\
\hline 25 & 0 & -1 & 1 & 0 & $5.656 \pm 0.04$ & 5.859 & $0.877 \pm 0.05$ & 0.911 & $2.969 \pm 0.05$ & 3.097 & $1.200 \pm 0.07$ & 1.345 \\
\hline 26 & 0 & 0 & 0 & 0 & $6.698 \pm 0.07$ & 6.835 & $1.645 \pm 0.03$ & 1.555 & $2.885 \pm 0.04$ & 2.517 & $1.640 \pm 0.05$ & 1.634 \\
\hline 27 & 0 & 0 & -1 & -1 & $6.379 \pm 0.03$ & 6.383 & $5.556 \pm 0.07$ & 5.556 & $2.542 \pm 0.06$ & 2.570 & $3.900 \pm 0.04$ & 3.900 \\
\hline 28 & -1 & 0 & 0 & -1 & $1.863 \pm 0.01$ & 1.863 & $0.513 \pm 0.01$ & 0.513 & $2.983 \pm 0.07$ & 2.983 & $1.800 \pm 0.01$ & 2.035 \\
\hline 29 & 0 & 0 & 1 & -1 & $7.042 \pm 0.02$ & 6.717 & $6.667 \pm 0.06$ & 6.667 & $3.454 \pm 0.05$ & 3.426 & $1.000 \pm 0.03$ & 1.000 \\
\hline
\end{tabular}

Measured values are medians of three repetitions \pm standard deviation. 
Table 3. The variables of the models and their significance after analysis of variance (ANOVA), for avocado peel's experimental results.

\begin{tabular}{|c|c|c|c|c|c|c|c|}
\hline \multicolumn{4}{|c|}{ Avocado Peel } & \multicolumn{4}{|c|}{ Avocado Peel } \\
\hline \multicolumn{2}{|c|}{$\begin{array}{c}\text { TPC } \\
\text { (mg GAE/g fw) }\end{array}$} & \multicolumn{2}{|c|}{$\begin{array}{c}\text { EEqV R } \\
\text { IC }_{50} \mathrm{DPPH}^{\bullet}(\mathrm{L})\end{array}$} & \multicolumn{2}{|c|}{$\begin{array}{c}\text { Ratio TPC } \\
(\mathrm{mg} \mathrm{GAE} / \mathrm{g} \mathrm{fw}) / \mathrm{min}\end{array}$} & \multicolumn{2}{|c|}{$\begin{array}{c}\text { Ratio EEqV R } \\
\text { IC }_{50} \mathrm{DPPH}^{\bullet}(\mathrm{L}) / \mathrm{min}\end{array}$} \\
\hline & $p$ Value & & $p$ Value & & $p$ Value & & $p$ Value \\
\hline Model & $<0.0001 *$ & Model & $<0.0001 *$ & Model & $<0.0001 *$ & Model & $<0.0001 *$ \\
\hline Variables & $p$ Value & Variables & $p$ Value & Variables & $p$ Value & Variables & $p$ Value \\
\hline $\mathrm{A}$ & 0.0318 * & A-TEMP. & 0.2881 & A-TEMP. & 0.0133 & A-TEMP. & 0.1446 \\
\hline B & $<0.0001 *$ & B-TIME & $<0.0001 *$ & B-TIME & $<0.0001 *$ & B-TIME & 0.0039 * \\
\hline $\mathrm{C}$ & $0.0002 *$ & C-POWER & 0.3122 & C-POWER & $0.0001^{*}$ & C-POWER & 0.2036 \\
\hline $\mathrm{D}$ & 0.2409 & D-RATIO & $0.0036^{*}$ & D-RATIO & $0.0132 *$ & D-RATIO & 0.1519 \\
\hline $\mathrm{AB}$ & 0.0009 * & $\mathrm{AD}$ & $<0.0001 *$ & $\mathrm{AB}$ & $<0.0001 *$ & AC & 0.7236 \\
\hline $\mathrm{AC}$ & 0.0002 * & $\mathrm{BD}$ & $0.0016^{*}$ & $\mathrm{AC}$ & $<0.0001^{*}$ & $\mathrm{AD}$ & $<0.0001^{*}$ \\
\hline $\mathrm{AD}$ & $<0.0001 *$ & $\mathrm{CD}$ & 0.1169 & AD & $<0.0001 *$ & $\mathrm{BD}$ & 0.0316 * \\
\hline $\mathrm{BD}$ & $0.0014 *$ & $\mathrm{~A}^{2}$ & 0.1413 & $\mathrm{BC}$ & $0.0490 *$ & $\mathrm{CD}$ & 0.1801 \\
\hline CD & 0.0279 * & $B^{2}$ & 0.0103 * & $\mathrm{BD}$ & $<0.0001 *$ & $\mathrm{~A}^{2}$ & 0.2709 \\
\hline $\mathrm{A}^{2}$ & 0.7879 & $C^{2}$ & 0.2281 & CD & 0.0103 * & $B^{2}$ & $0.0030^{*}$ \\
\hline $\mathrm{B}^{2}$ & $<0.0001 *$ & $\mathrm{D}^{2}$ & 0.5418 & $\mathrm{~B}^{2}$ & $<0.0001 *$ & $C^{2}$ & 0.0128 * \\
\hline$C^{2}$ & 0.0006 * & $A^{2} D$ & 0.0002 * & $C^{2}$ & $0.0019 *$ & $\mathrm{D}^{2}$ & 0.0590 * \\
\hline $\mathrm{D}^{2}$ & 0.073 & $\mathrm{AD}^{2}$ & 0.0185 * & $\mathrm{D}^{2}$ & $<0.0001 *$ & $A^{2} C$ & 0.4777 \\
\hline$A^{2} B$ & 0.0698 & $C^{2} D$ & $<0.0001 *$ & $\mathrm{AB}^{2}$ & $<0.0001 *$ & $A^{2} D$ & 0.0084 * \\
\hline$A^{2} D$ & 0.0264 * & $\mathrm{CD}^{2}$ & $0.0006^{*}$ & $B^{2} D$ & $<0.0001 *$ & $A C^{2}$ & 0.2850 \\
\hline $\mathrm{AB}^{2}$ & 0.0103 * & $A^{2} D^{2}$ & 0.0097 * & $\mathrm{BD}^{2}$ & $<0.0001 *$ & $\mathrm{AD}^{2}$ & 0.1980 \\
\hline $\mathrm{AD}^{2}$ & 0.3003 & $C^{2} D^{2}$ & $<0.0001 *$ & $C^{2} D$ & 0.3247 & $C^{2} \mathrm{D}$ & $<0.0001 *$ \\
\hline$C^{2} D$ & 0.0068 * & & & $\mathrm{CD}^{2}$ & 0.5370 & $\mathrm{CD}^{2}$ & $0.0162 *$ \\
\hline \multirow[t]{2}{*}{$\mathrm{A}^{2} \mathrm{D}^{2}$} & $0.003 *$ & & & $C^{2} D^{2}$ & 0.0001 * & $A^{2} C^{2}$ & 0.0189 * \\
\hline & & & & & & $C^{2} D^{2}$ & 0.0004 * \\
\hline \multirow{3}{*}{$\begin{array}{l}\text { Lack of } \\
\text { fitting }\end{array}$} & 0.6055 & & 0.9780 & & 0.9214 & & 0.8839 \\
\hline & Not & & Not & & Not & & Not \\
\hline & significant & & significant & & significant & & Significant \\
\hline$R^{2}$ & 0.9857 & \multicolumn{2}{|c|}{0.9906} & & 0.9974 & & 0.99 \\
\hline Adj. $R^{2}$ & 0.9555 & \multicolumn{2}{|c|}{0.9761} & & 0.9918 & & 0.9649 \\
\hline
\end{tabular}

${ }^{*} p<0.05$ was considered to be statistically significant A; Extraction temperature $\left({ }^{\circ} \mathrm{C}\right), \mathrm{B}$ : Extraction time (min), C: Microwave power $(\mathrm{W}), \mathrm{D}$ : Ratio of water to avocado by-product (\%).

Table 4. The models' variables and their significance after analysis of variance (ANOVA) for avocado seeds' experimental results.

\begin{tabular}{|c|c|c|c|c|c|c|c|}
\hline \multicolumn{4}{|c|}{ Avocado Seed } & \multicolumn{4}{|c|}{ Avocado Seed } \\
\hline \multicolumn{2}{|c|}{$\begin{array}{c}\text { TPC } \\
(\mathrm{mg} \mathrm{GAE} / \mathrm{g} \mathrm{fw})\end{array}$} & \multicolumn{2}{|c|}{$\begin{array}{c}\text { EEqV R } \\
\text { IC }_{50} \mathrm{DPPH}^{\bullet}(\mathrm{L})\end{array}$} & \multicolumn{2}{|c|}{$\begin{array}{c}\text { Ratio TPC } \\
(\mathrm{mg} \mathrm{GAE} / \mathrm{g} \mathrm{fw}) / \mathrm{min}\end{array}$} & \multicolumn{2}{|c|}{$\begin{array}{c}\text { Ratio EEqV R } \\
\text { IC }_{50} \mathrm{DPPH}^{\bullet}(\mathrm{L}) / \mathrm{min}\end{array}$} \\
\hline & $p$ Value & & $p$ Value & & $p$ Value & & $p$ Value \\
\hline Model & $<0.0001 *$ & Model & $<0.0001 *$ & Model & $<0.0009 *$ & Model & $<0.0001$ * \\
\hline Variables & $p$ Value & Variables & $p$ Value & Variables & $p$ Value & Variables & $p$ Value \\
\hline A & $0.0227 *$ & A & 0.1220 & $\mathrm{~A}$ & 0.0507 & A & 0.8792 \\
\hline B & $<0.0001 *$ & B & $<0.0001 *$ & B & 0.0009 * & B & 0.8098 \\
\hline $\mathrm{C}$ & $0.0192 *$ & $\mathrm{C}$ & $0.0004 *$ & $\mathrm{C}$ & $0.0371 *$ & $\mathrm{C}$ & 0.1483 \\
\hline $\mathrm{D}$ & 0.0048 * & $\mathrm{D}$ & 0.4145 & $\mathrm{D}$ & $0.0129 *$ & $\mathrm{D}$ & 0.9703 \\
\hline $\mathrm{AB}$ & 0.0143 * & $\mathrm{AC}$ & 0.1145 & $\mathrm{AB}$ & 0.0816 & $\mathrm{AC}$ & 0.0037 * \\
\hline $\mathrm{AC}$ & $0.0002 *$ & $\mathrm{AD}$ & 0.0197 * & $\mathrm{AC}$ & $0.0013 *$ & $\mathrm{AD}$ & 0.0031 * \\
\hline
\end{tabular}


Table 4. Cont.

\begin{tabular}{|c|c|c|c|c|c|c|c|}
\hline \multicolumn{4}{|c|}{ Avocado Seed } & \multicolumn{4}{|c|}{ Avocado Seed } \\
\hline \multicolumn{2}{|c|}{$\begin{array}{c}\text { TPC } \\
(\mathrm{mg} \mathrm{GAE} / \mathrm{g} \mathrm{fw})\end{array}$} & \multicolumn{2}{|c|}{$\begin{array}{c}\text { EEqV R } \\
\text { IC }_{50} \mathrm{DPPH}^{\bullet}(\mathrm{L})\end{array}$} & \multicolumn{2}{|c|}{$\begin{array}{c}\text { Ratio TPC } \\
(\mathrm{mg} \mathrm{GAE} / \mathrm{g} \mathrm{fw}) / \mathrm{min}\end{array}$} & \multicolumn{2}{|c|}{$\begin{array}{c}\text { Ratio EEqV R } \\
\text { IC }_{50} \mathrm{DPPH}^{\bullet}(\mathrm{L}) / \mathrm{min}\end{array}$} \\
\hline & $p$ Value & & $p$ Value & & $p$ Value & & $p$ Value \\
\hline Model & $<0.0001$ * & Model & $<0.0001 *$ & Model & $<0.0009 *$ & Model & $<0.0001 *$ \\
\hline Variables & $p$ Value & Variables & $p$ Value & Variables & $p$ Value & Variables & $p$ Value \\
\hline $\mathrm{AD}$ & 0.0003 * & $\mathrm{BC}$ & 0.0064 * & $\mathrm{AD}$ & 0.0018 * & $\mathrm{CD}$ & $<0.0001$ * \\
\hline $\mathrm{BC}$ & 0.0329 * & $\mathrm{CD}$ & 0.0703 & $\mathrm{BC}$ & 0.0002 * & $\mathrm{A}^{2}$ & 0.1139 \\
\hline $\mathrm{BD}$ & 0.4689 & $\mathrm{~A}^{2}$ & 0.5569 & $\mathrm{BD}$ & 0.3183 & $\mathrm{~B}^{2}$ & 0.0024 * \\
\hline $\mathrm{A}^{2}$ & 0.0256 * & $C^{2}$ & 0.7035 & $\mathrm{~A}^{2}$ & 0.0964 & $C^{2}$ & 0.1839 \\
\hline $\mathrm{B}^{2}$ & $<0.0001 *$ & $\mathrm{D}^{2}$ & 0.7781 & $\mathrm{~B}^{2}$ & $<0.0001 *$ & $\mathrm{D}^{2}$ & 0.6190 \\
\hline$C^{2}$ & 0.4497 & $A^{2} D$ & 0.0324 * & $C^{2}$ & 0.1660 & $A^{2} C$ & 0.0230 * \\
\hline $\mathrm{D}^{2}$ & 0.9014 & $C^{2} \mathrm{D}$ & 0.8975 & $\mathrm{D}^{2}$ & 0.3659 & $A^{2} D$ & 0.0255 * \\
\hline$A^{2} C$ & 0.3652 & $\mathrm{CD}^{2}$ & 0.3007 & $A^{2} B$ & 0.0544 * & $A C^{2}$ & 0.0174 * \\
\hline$A^{2} D$ & 0.0180 * & $C^{2} D^{2}$ & 0.1615 & $A^{2} C$ & 0.4081 & $C^{2} D$ & $0.0011 *$ \\
\hline $\mathrm{AC}^{2}$ & 0.5203 & & & $A^{2} D$ & 0.0353 * & $\mathrm{CD}^{2}$ & $<0.0001$ * \\
\hline $\mathrm{AD}^{2}$ & 0.9120 & & & $\mathrm{AB}^{2}$ & 0.4393 & $C^{2} D^{2}$ & 0.0003 * \\
\hline$B^{2} C$ & 0.0891 & & & $A C^{2}$ & 0.6226 & & \\
\hline$B^{2} D$ & 0.0252 * & & & $\mathrm{B}^{2} \mathrm{C}$ & 0.0020 * & & \\
\hline$A^{2} C^{2}$ & 0.0009 * & & & $B^{2} D$ & 0.0260 * & & \\
\hline \multirow[t]{5}{*}{$\mathrm{A}^{2} \mathrm{D}^{2}$} & 0.0055 * & & & $\mathrm{BC}^{2}$ & 0.1076 & & \\
\hline & & & & $\mathrm{BD}^{2}$ & - & & \\
\hline & & & & $A^{2} B^{2}$ & 0.0111 * & & \\
\hline & & & & $A^{2} C^{2}$ & 0.0704 & & \\
\hline & & & & $B^{2} C^{2}$ & - & & \\
\hline \multirow{3}{*}{$\begin{array}{l}\text { Lack of } \\
\text { fitting }\end{array}$} & 0.8247 & & 0.9155 & & 0.8760 & & 0.9542 \\
\hline & Not & & Not & & Not & & Not \\
\hline & significant & & significant & & significant & & significant \\
\hline$R^{2}$ & 0.9909 & & 0.9234 & & 0.9919 & & 0.9773 \\
\hline Adj. $R^{2}$ & 0.9636 & & 0.8350 & & 0.9545 & & 0.9423 \\
\hline
\end{tabular}

${ }^{*} p<0.05$ was considered to be statistically significant A; Extraction temperature $\left({ }^{\circ} \mathrm{C}\right), \mathrm{B}$ : Extraction time (min), C: Microwave power $(\mathrm{W}), \mathrm{D}$ : Ratio of water to avocado by-product (\%).

Table 5. Prediction equations for each response derived from the application of multiple regression analysis on experimental data using the Design Expert v12 software package.

\begin{tabular}{|c|c|c|c|c|c|c|c|c|c|c|c|c|c|c|}
\hline \multicolumn{4}{|c|}{ TPC } & \multicolumn{4}{|c|}{ EEqV R IC50 DPPH• } & \multicolumn{4}{|c|}{ RATIO TPC } & \multicolumn{3}{|c|}{ RATIO EEqV R IC50 DPPH ${ }^{\bullet}$} \\
\hline $\mathbf{A P}$ & & AS & & AP & & AS & & $\mathbf{A P}$ & & AS & & AP & AS & \\
\hline $\begin{array}{c}= \\
+6.84\end{array}$ & & $\begin{array}{c}= \\
+2.52\end{array}$ & & $\begin{array}{c}= \\
+0.106\end{array}$ & & & & $\begin{array}{c}= \\
+1.55\end{array}$ & & +0.0387 & & $\begin{array}{c}= \\
+0.024\end{array}$ & +150.87 & \\
\hline+0.5182 & A & +0.4115 & A & +0.0055 & A & -0.2089 & A & +0.0900 & A & +0.0060 & A & $+0.0033 \mathrm{~A}$ & +2.66 & A \\
\hline+1.42 & B & +1.68 & B & -0.0673 & B & -1.07 & B & +0.5275 & B & -0.0162 & B & $-0.0047 \quad \mathrm{~B}$ & +3.44 & B \\
\hline+0.7017 & $\mathrm{C}$ & +0.4281 & $\mathrm{C}$ & +0.0113 & $\mathrm{C}$ & -0.7300 & C & +0.0854 & $\mathrm{C}$ & +0.0066 & $\mathrm{C}$ & $+0.0028 \quad \mathrm{C}$ & -37.59 & $\mathrm{C}$ \\
\hline-0.2562 & $\mathrm{D}$ & -0.5740 & $\mathrm{D}$ & +0.0078 & $\mathrm{D}$ & -0.1844 & D & +0.4206 & $\mathrm{D}$ & -0.0088 & $\mathrm{D}$ & $+0.0032 \quad \mathrm{D}$ & -0.9210 & $\mathrm{D}$ \\
\hline-0.9922 & $\mathrm{AB}$ & +0.4583 & $\mathrm{AB}$ & -0.0467 & $\mathrm{AB}$ & +0.3700 & $\mathrm{AC}$ & -1.44 & $\mathrm{AD}$ & +0.0051 & $\mathrm{AB}$ & $-0.0007 \mathrm{AC}$ & +88.66 & $\mathrm{AC}$ \\
\hline+1.25 & $\mathrm{AC}$ & -0.9896 & $\mathrm{AC}$ & +0.0192 & $\mathrm{AC}$ & +0.5813 & $\mathrm{AD}$ & +0.4723 & $\mathrm{BD}$ & -0.0152 & $\mathrm{AC}$ & $-0.0222 \mathrm{AD}$ & +91.17 & $\mathrm{AD}$ \\
\hline-2.49 & $\mathrm{AD}$ & -0.9135 & $\mathrm{AD}$ & -0.0383 & $\mathrm{AD}$ & +0.7100 & $\mathrm{BC}$ & +0.1940 & $\mathrm{CD}$ & -0.0141 & $\mathrm{AD}$ & $+0.0053 \mathrm{BD}$ & +185.00 & CD \\
\hline+0.9271 & $\mathrm{BD}$ & -0.3750 & $\mathrm{BC}$ & -0.0058 & $\mathrm{BC}$ & +0.4313 & $\mathrm{CD}$ & -0.1761 & $\mathrm{~A}^{2}$ & -0.0218 & $\mathrm{BC}$ & $+0.0030 \mathrm{CD}$ & +34.74 & $\mathrm{~A}^{2}$ \\
\hline+0.5344 & $\mathrm{CD}$ & -0.1083 & $\mathrm{BD}$ & +0.0269 & $\mathrm{BD}$ & +0.1057 & $\mathrm{~A}^{2}$ & -0.3432 & $\mathrm{~B}^{2}$ & -0.0026 & $\mathrm{BD}$ & $+0.0023 \mathrm{~A}^{2}$ & -79.16 & $\mathrm{~B}^{2}$ \\
\hline+0.0551 & $\mathrm{~A}^{2}$ & -0.5583 & $\mathrm{~A}^{2}$ & +0.0082 & $\mathrm{CD}$ & +0.0828 & $C^{2}$ & +0.1419 & $C^{2}$ & +0.0067 & $\mathrm{~A}^{2}$ & $-0.0083 \mathrm{~B}^{2}$ & +33.42 & $C^{2}$ \\
\hline-1.13 & $\mathrm{~B}^{2}$ & +1.17 & $\mathrm{~B}^{2}$ & +0.0266 & $\mathrm{~B}^{2}$ & +0.0612 & $\mathrm{D}^{2}$ & -0.1003 & $\mathrm{D}^{2}$ & +0.0260 & $\mathrm{~B}^{2}$ & $+0.0091 C^{2}$ & +12.06 & $\mathrm{D}^{2}$ \\
\hline+0.8718 & $C^{2}$ & -0.1104 & $C^{2}$ & +0.0107 & $C^{2}$ & +0.7406 & $A^{2} D$ & -0.8609 & $A^{2} D$ & -0.0037 & $C^{2}$ & $+0.0044 \quad \mathrm{D}^{2}$ & -90.29 & $A^{2} C$ \\
\hline-0.4037 & $\mathrm{D}^{2}$ & +0.0177 & $\mathrm{D}^{2}$ & -0.0277 & $\mathrm{D}^{2}$ & +0.0406 & $C^{2} D$ & +0.3858 & $\mathrm{AD}^{2}$ & +0.0023 & $\mathrm{D}^{2}$ & $-0.0021 \mathrm{~A}^{2} \mathrm{C}$ & +88.29 & $A^{2} D$ \\
\hline
\end{tabular}


Table 5. Cont.

\begin{tabular}{|c|c|c|c|c|c|c|c|}
\hline \multicolumn{2}{|c|}{ TPC } & \multicolumn{2}{|c|}{ EEqV R IC50 DPPH・ } & \multicolumn{2}{|c|}{ RATIO TPC } & \multicolumn{2}{|c|}{ RATIO EEqV R IC50 DPPH ${ }^{\bullet}$} \\
\hline AP & AS & AP & AS & AP & AS & AP & AS \\
\hline $\begin{array}{ll}-0.5143 & \mathrm{~A}^{2} \mathrm{~B} \\
+0.7656 & \mathrm{~A}^{2} \mathrm{D} \\
+0.9323 & \mathrm{AB}^{2} \\
-0.3172 & \mathrm{AD}^{2} \\
+1.01 & \mathrm{C}^{2} \mathrm{D} \\
-1.42 & \mathrm{~A}^{2} \mathrm{D}^{2}\end{array}$ & $\begin{array}{l}-0.1937 \mathrm{~A}^{2} \mathrm{C} \\
-0.6146 \mathrm{~A}^{2} \mathrm{D} \\
-0.1354 \mathrm{AC}^{2} \\
-0.0229 \mathrm{AD}^{2} \\
+0.3948 \mathrm{~B}^{2} \mathrm{C} \\
+0.5677 \mathrm{~B}^{2} \mathrm{D} \\
+1.56 \mathrm{~A}^{2} \mathrm{C}^{2} \\
+1.12 \mathrm{~A}^{2} \mathrm{D}^{2}\end{array}$ & $\begin{array}{l}+0.0455 \mathrm{AB}^{2} \\
-0.0283 \mathrm{~B}^{2} \mathrm{D} \\
+0.0259 \mathrm{BD}^{2} \\
+0.0038 \mathrm{C}^{2} \mathrm{D} \\
+0.0020 \mathrm{CD}^{2} \\
+0.0262 \mathrm{C}^{2} \mathrm{D}^{2}\end{array}$ & $\begin{array}{l}-0.2888 \mathrm{CD}^{2} \\
+0.5279 \mathrm{C}^{2} \mathrm{D}^{2}\end{array}$ & $\begin{array}{ll}-2.57 & \mathrm{C}^{2} \mathrm{D} \\
+0.6642 & \mathrm{CD}^{2} \\
+0.7120 & \mathrm{~A}^{2} \mathrm{D}^{2} \\
+2.37 & \mathrm{C}^{2} \mathrm{D}^{2}\end{array}$ & $\begin{array}{l}+0.0083 \mathrm{~A}^{2} \mathrm{~B} \\
-0.0030 \mathrm{~A}^{2} \mathrm{C} \\
-0.0095 \mathrm{~A}^{2} \mathrm{D} \\
-0.0028 \mathrm{AB}^{2} \\
-0.0017 \mathrm{AC}^{2} \\
+0.0195 \mathrm{~B}^{2} \mathrm{C} \\
+0.0103 \mathrm{~B}^{2} \mathrm{D} \\
+0.0065 \mathrm{BC}^{2}\end{array}$ & $\begin{array}{l}-0.0100 \mathrm{~A}^{2} \mathrm{D} \\
-0.0033 \mathrm{AC}^{2} \\
+0.0040 \mathrm{AD}^{2} \\
-0.0363 \mathrm{C}^{2} \mathrm{D} \\
+0.0087 \mathrm{CD}^{2} \\
-0.0119 \mathrm{~A}^{2} \mathrm{C}^{2} \\
+0.0236 \mathrm{C}^{2} \mathrm{D}^{2}\end{array}$ & $\begin{array}{l}-82.80 \mathrm{AC}^{2} \\
-150.58 \mathrm{C}^{2} \mathrm{D} \\
-266.26 \mathrm{CD}^{2} \\
+221.75 \mathrm{C}^{2} \mathrm{D}^{2}\end{array}$ \\
\hline
\end{tabular}

A, B, C and D are the coded extraction parameters: A: Extraction temperature $\left({ }^{\circ} \mathrm{C}\right)$; B: Extraction time (min); C: Microwave power $(\mathrm{W}) ; \mathrm{D}$ : Ratio of water to avocado by-product (\%).

\subsection{Optimization of Avocado By-Products Vacuum Microwave-Assisted Aqueous Extraction}

Using Design Expert v12 statistical and optimization software, the optimal conditions for the extraction parameters were defined in order to optimize the two selected responses taking into account the operational extraction cost. Thus, setting as target the maximum rate of receiving extracts with the higher TPC/g and EEqRVIC ${ }_{50} \mathrm{DPPH}^{\bullet}$ the independent variables received, respectively, and the selected program solutions with desirability $=1000$ are presented in Table 6.

Table 6. Predicted responses values at the optimum conditions for rate of total phenolic content (RTPC) and the rate of Extract Equivalent Volume $(\mathrm{EEqV})$ at Reference $\mathrm{IC}_{50}$ of $\mathrm{DPPH}^{\bullet}$ radical scavenging activity equal to $100 \mathrm{mg} / \mathrm{L}\left(\mathrm{REEqV} \mathrm{RIC}_{50} \mathrm{DPPH}^{\bullet}\right)$.

\begin{tabular}{ccccccc}
\hline & \multicolumn{6}{c}{ Independent Variables } \\
\hline A & B & C & D & $\begin{array}{c}\text { RTPC (mg GAE/g } \\
\text { fw)/min }\end{array}$ & $\begin{array}{c}\text { REEqV RIC }_{\mathbf{5 0}} \\
\text { DPPH }^{\bullet} \text { L/min }\end{array}$ \\
\hline AP & 79.64 & 11.89 & 5708.04 & 16.45 & 0.352 & \\
\hline AP & 78.11 & 11.75 & 5699.10 & 10.02 & & 0.104 \\
\hline AS & 43.90 & 10.18 & 5549.08 & 38.73 & 0.124 & 0.045 \\
\hline
\end{tabular}

A: Extraction temperature $\left({ }^{\circ} \mathrm{C}\right)$; B: Extraction time $(\mathrm{min}) ; \mathrm{C}$ : Microwave power $(\mathrm{W})$; D: Ratio of water to avocado by-product (\%).

\subsection{Effects of Independent Variables and Their Interaction}

The effects of regression equations, schematic representations of three-dimensional response surfaces and two-dimensional contour plots are illustrated in Figure 1, Figure 2, Figure 3, Figure 4. The correlation between independent and dependent variables and the interactions between them were visualized using 2-3D plots by using the $\mathrm{z}$-axis while retaining the other two variables at their zero level. Different shapes of the contour plots suggest various interactions between variables. Circular contour plots present negligible correlations between the related variables. The elliptical contour revealed substantially different interactions between the related variables [36]. Microwave temperature (A) and power (C) parameters have reported that they are interrelated. In more detail, it was observed that as microwave power and extraction temperature increase the yield extraction is also increased [23]. Due to reduced viscosity and surface tension, the solvent strength increased at high temperatures, promoting solvent solubilization and increasing hydration of the extracting materials [37]. The significance of the interaction between these independent parameters also presented in the ANOVA (Tables 3 and 4) (Figures 2a, 3b and 4a). Segovia et al. [18] evaluated the effect of ultrasound $(0-104 \mathrm{~W})$ and temperature range $\left(20-60^{\circ} \mathrm{C}\right)$ on the effective extraction of polyphenols from avocado seeds dissolved in water. According to their results, the increase of 
the ultrasonic power and the extraction temperature resulted in extracts with higher polyphenol content and antioxidant capacity reaching an optimum at their higher tested temperature of $60{ }^{\circ} \mathrm{C}$ and presented also a linear relationship between the antioxidant activity and the TPC.

However, studies have reported that the extraction efficacy increases when extraction temperature rises until the optimal temperature is reached and then begins to decrease as the temperature rises further. This phenomenon was presented in the extraction of AS, as can been seen in Figure 3a. It was also observed that differences in extraction temperature may affect the antioxidant ability of the polyphenolic compounds [38]. Furthermore, when VMAAE was evaluated under vacuum system like the system of MAC-75 (Milestone Technologies Inc., Sorisole, Italy) industrial microwave extractor with high power efficiency, the temperature of the boiling point decreased as a result of the vacuum procedure, resulting in better extraction efficiency by the desorption of solutes from active sites within the extracted mixture [28].

From the ANOVA, it is clearly demonstrated that the extraction time (B) affects significantly the efficacy of the VMAAE procedure from the point of operational cost. Thus, the operational cost in this study was estimated on the basis of the industrial extraction rate. The only exception to this was the ratio of $\mathrm{EEqV} \mathrm{R} \mathrm{IC}_{50} \mathrm{DPPH}^{\bullet}$ (L) from AS, probably because of the different moisture concentration and the different extracts phenolic profile [8]. Extraction time in MAE has been reported to be short in contrast with the traditional techniques used so far (stirring and maceration) and typically ranges between a few minutes and half an hour, preventing potential degradation and oxidation of bioactive compounds [24]. As represented in Table 6, a period of $11.75 \mathrm{~min}$ and $10.18 \mathrm{~min}$ were observed in order to achieve the optimal extraction time of AP and AS, respectively. This observation is based on the extraction rate of $\mathrm{REEqVR} \mathrm{IC}_{50} \mathrm{DPPH}^{\bullet}$, which was similar to the optimal extraction time $(12.127 \mathrm{~min})$ used for the extraction of pomegranate peels using the same methodology [28]. The extraction time used in this study is shorter than the required extraction time needed for the extraction of by-products of dry avocados as described by Calderón-Oliver et al., [35]. The two aforementioned methods also require shorter time compared with the process as described by Morais et al. [39] where $4 \mathrm{~h}$ extraction time is required in order to achieve production of 1.81 and $1.5 \mathrm{mg}$ GAE/g from fresh peel and seeds using methanol as a solvent.

The interaction among the extraction time (B) and the extraction temperature (A) were statistically significant for the extraction of the two by-products based on ANOVA analysis on TPC, EEqVR $\mathrm{IC}_{50} \mathrm{DPPH}^{\bullet}$ and their ratio of TPC (except for REEqVR IC 50 $\mathrm{DPPH}^{\bullet}$ of AP) (Tables 3 and 4). Additionally, as shown in Table 3 , the interaction between extraction time (B) and the ratio of water to AP (\%) (D) (Figures 1c and 2c) were found to be significant according to ANOVA. This can be explained from the fact that the volume of the solvent must be adequate in order to ensure that the extracted plant material is submerged in the water during the microwave process and this has also been presented as significant by a similar research work [32].Additionally in our study, it was observed in the first $10-12 \mathrm{~min}$ and in a ratio of $10-16.45 \%$ the achievement of the excellent extraction flows from AP with the highest TPC and antioxidant activities while for AS the ratio needs to be higher (37.65-38.73\%). This observations is probably explained by the different moisture contain [40]. The fast and effective extraction of phenolic compounds from avocado by-products by the MAE may be explained by the rapid generation of heat by microwave radiation, which induces the degradation of the cellular matrix and increases the release of phenolic compounds and thus antioxidant activity [41,42]. On the other side the combinations of high temperatures with high extraction time increases the thermal stress of polyphenols leading to their degradation. This phenomenon is presented in Figure 3a and is in line with the study of Trujillo et al. [43] who optimized the AP extraction with a combined method of UAE and MAE using laboratory extractors and concluded that the optimal extraction temperature was $15 \mathrm{~min}$ at $60^{\circ} \mathrm{C}$ with UAE and $60 \mathrm{~s}$ with MAE. AP extraction increases after the optimal extraction time. Decrease of the polyphenolic contents of the extracts may be caused by their degradation. The 
higher optimal temperature in our study is probably due to higher extracted volumes in the industrial-scale extractor. Furthermore, a possible explanation in difference observed between the optimal temperatures in AP and AS is probably due to different phenolic profile and the degradation of certain phenolic compounds [44].

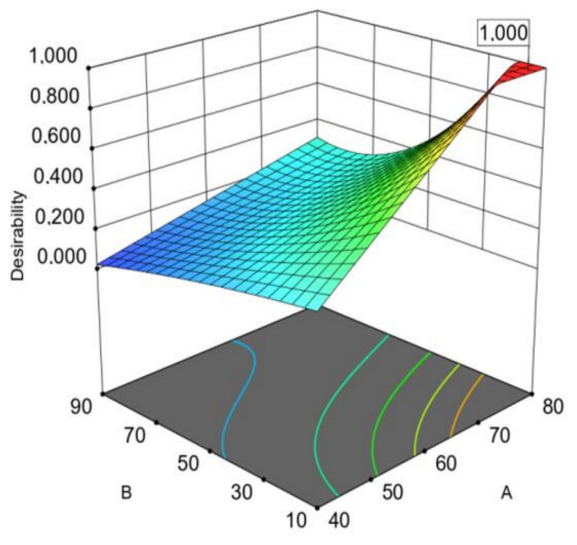

(a)

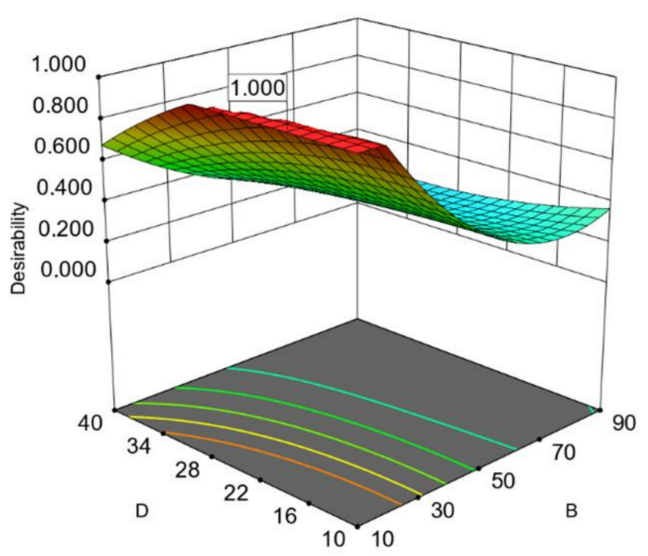

(c)

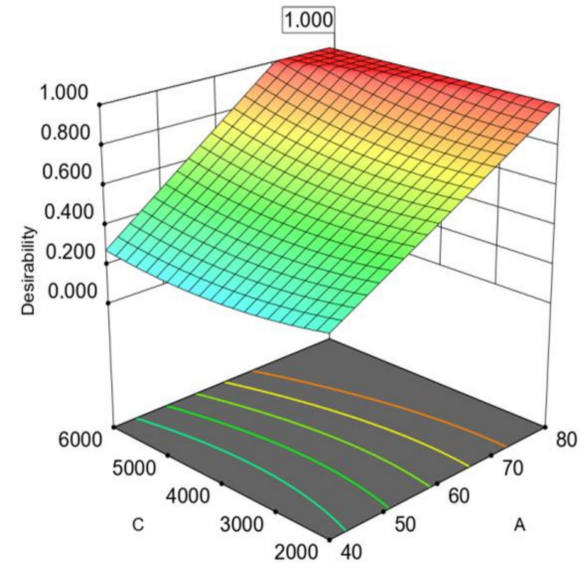

(b)

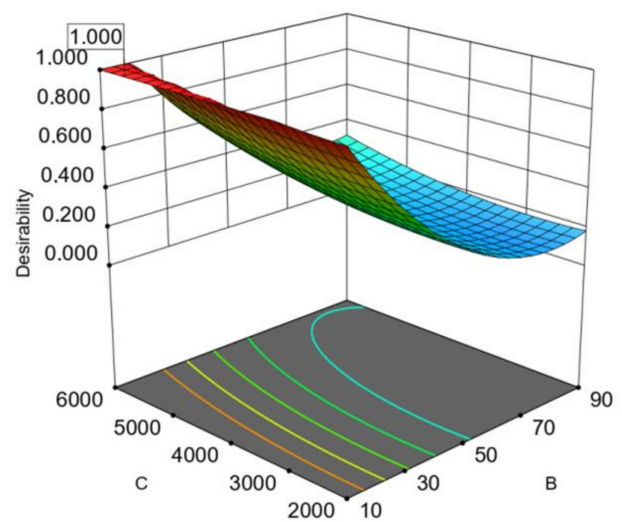

(d)

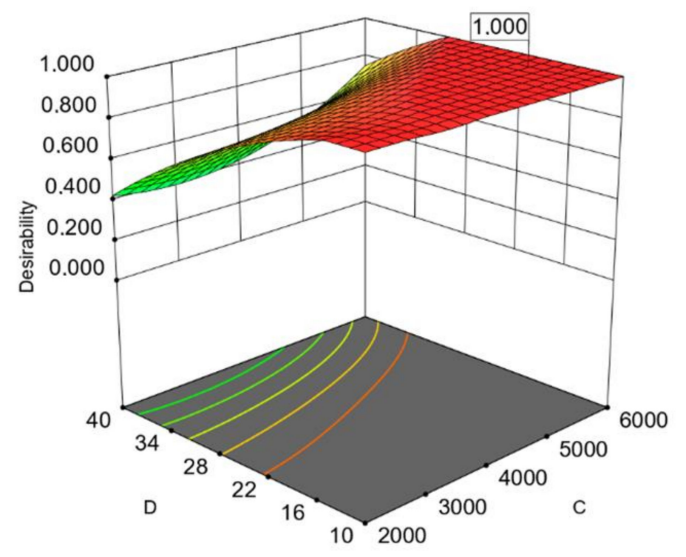

(e)

Figure 1. Response surface (3D) and (2D) contour plots presenting the interactions of the most significant extraction parameters based on operational costs for RTPC of avocado peel: (a) extraction temperature (A) and extraction time (B); (b) extraction temperature (A) and microwave power (C); (c) extraction time (B) and water to solid ratio (D); (d) extraction time (B) and microwave power $(C)$; (e) microwave power $(C)$ and water to solid ratio (D). 


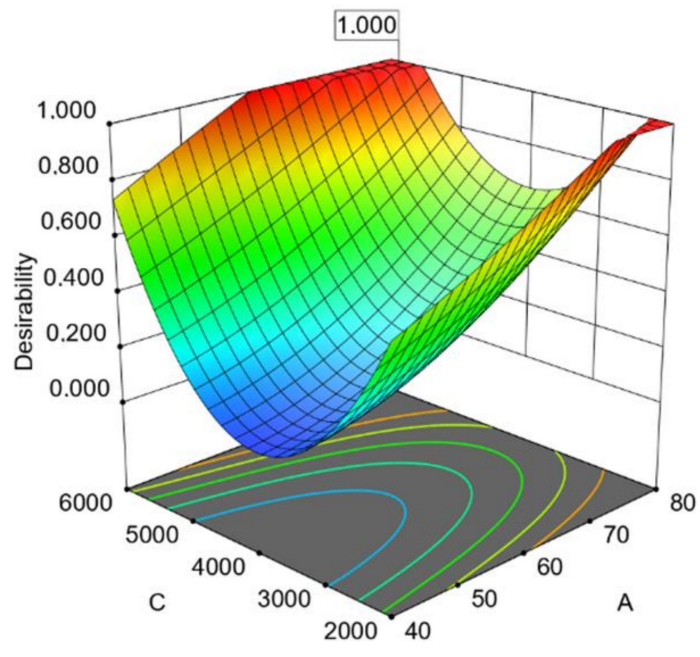

(a)

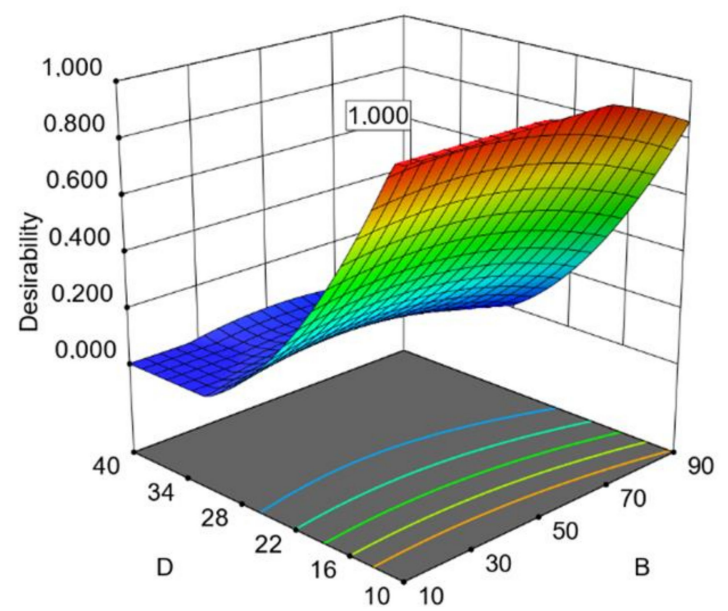

(c)

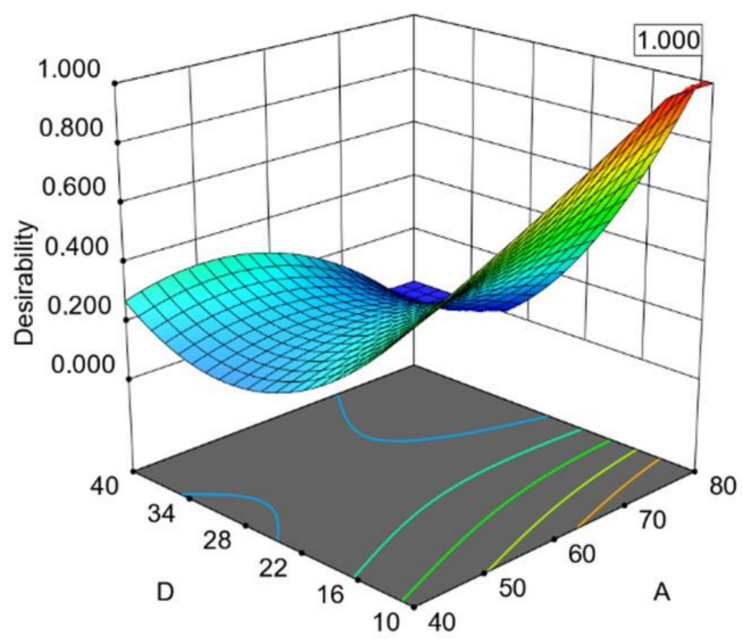

(b)

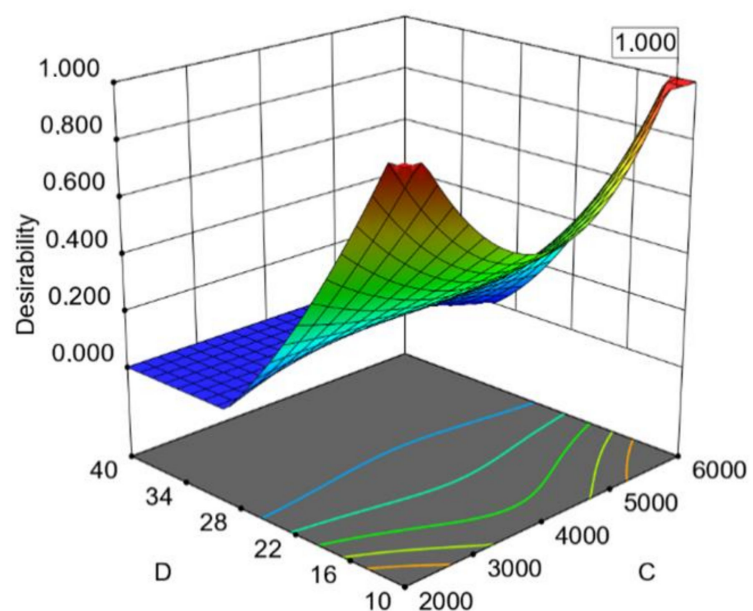

(d)

Figure 2. Response surface (3D) with (2D) contour plots presenting the interactions extraction parameters of avocado peel based on operational costs for the REEqVR IC $50 \mathrm{DPPH}^{\bullet}$ : (a) extraction temperature (A) and microwave power (C); (b) extraction temperature (A) and water to solid ratio (D); (c) extraction time (B) and water to solid ratio (D); (d) microwave power $(\mathrm{C})$ and water to solid ratio (D). 


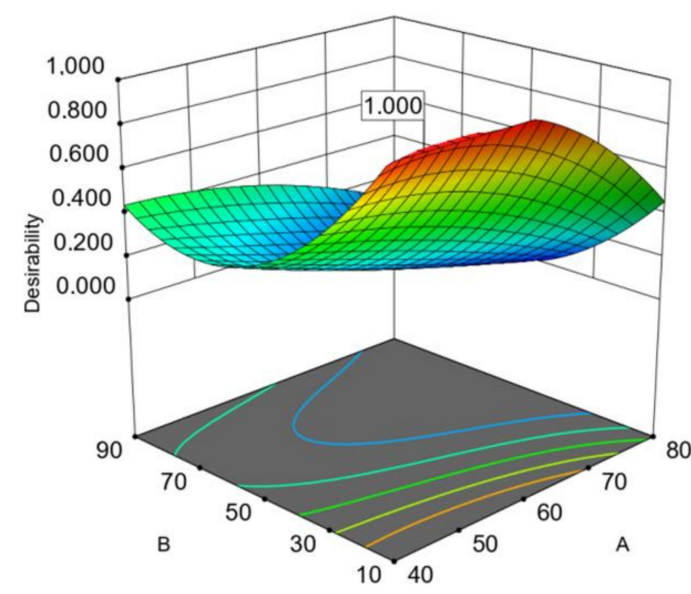

(a)

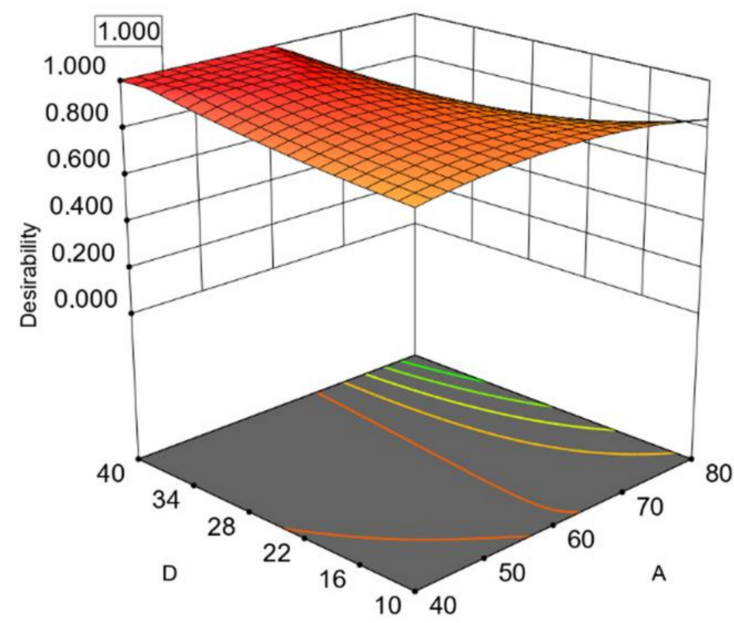

(c)

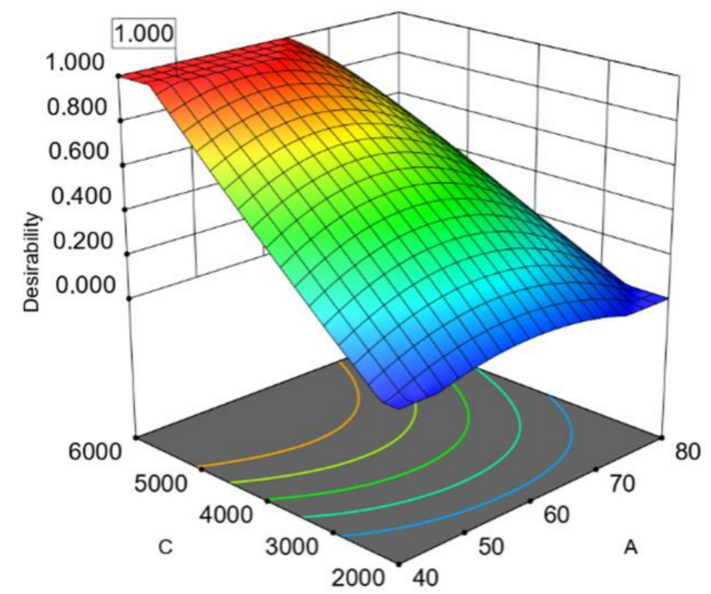

(b)

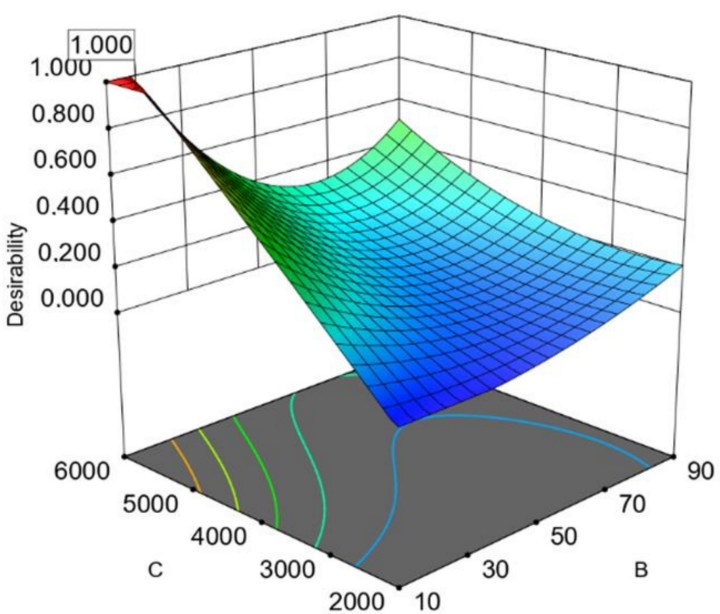

(d)

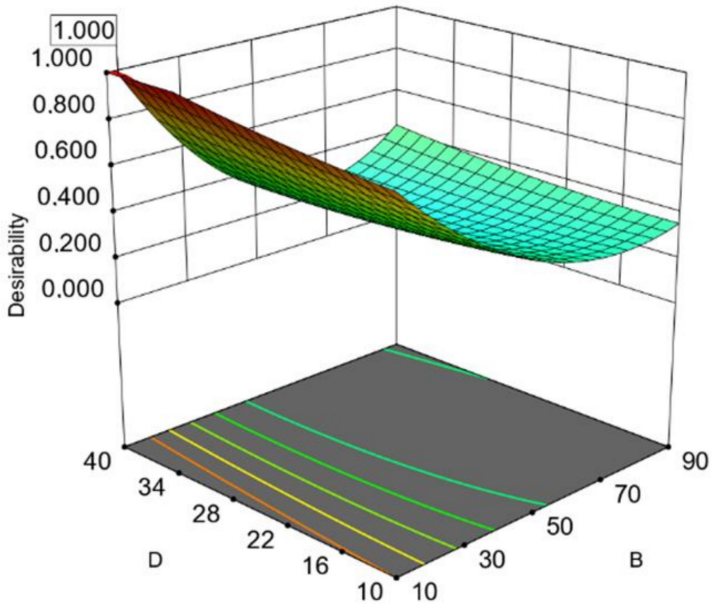

(e)

Figure 3. Response surface (3D) with (2D) contour plots presenting the interactions of the most significant extraction parameters of avocado seeds based on operational costs for RTPC: (a) extraction temperature (A) and extraction time (B); (b) extraction temperature (A) and microwave power (C); (c) extraction temperature (A) and water to solid ratio(D); (d) extraction time (B) and microwave power (C); (e) extraction time (B) and water to solid ratio (D). 


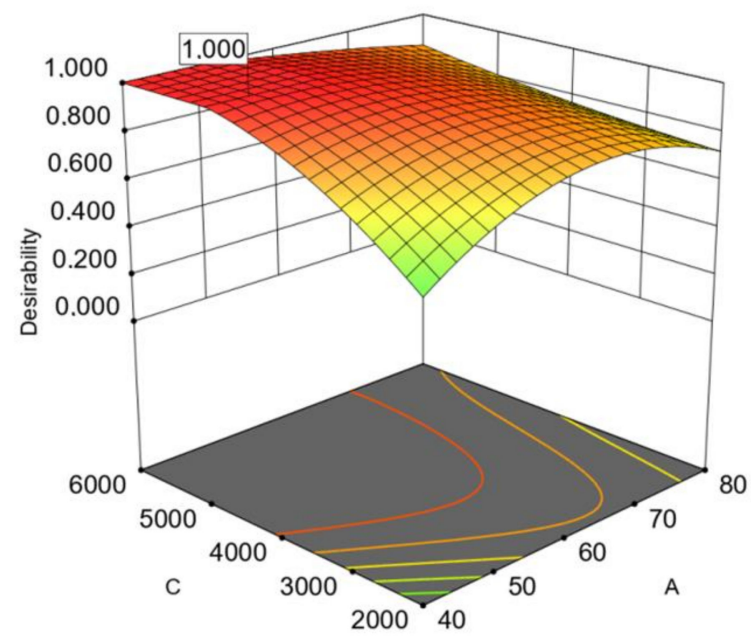

(a)

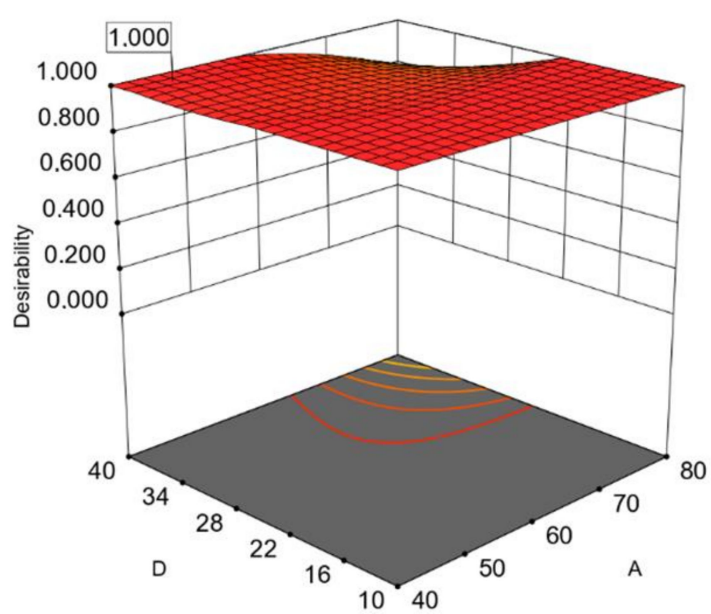

(b)

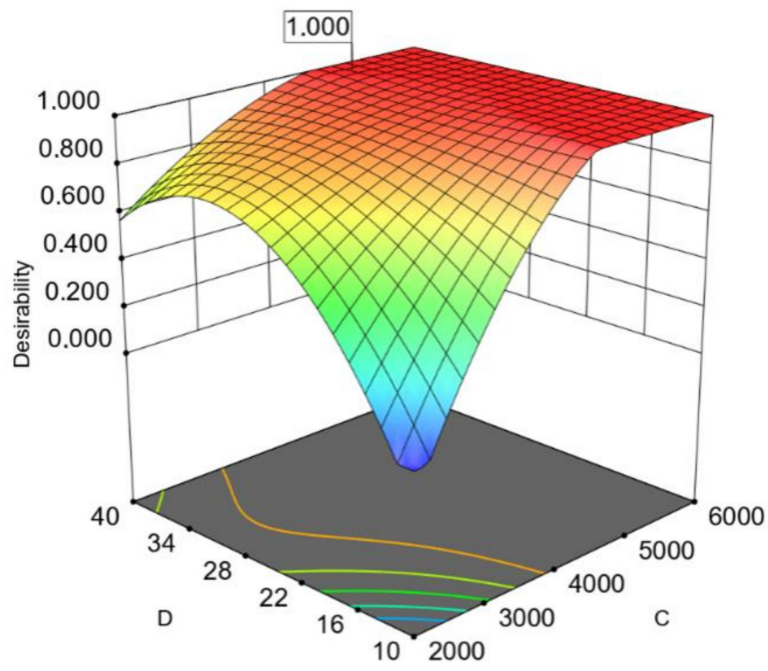

(c)

Figure 4. Response surface (3D) with (2D) contour plots presenting the interactions of the most significant extraction parameters of avocado seeds based on operational costs for RTPC: (a) extraction temperature (A) and microwave power (C); (b) extraction temperature (A) and water to solid ratio (D); (c) microwave power (C) and water to solid ratio (D).

The interaction of extraction time (B) with extraction power (C) were estimated as significant from ANOVA for the rate of total phenolic content of avocado peel and seeds as presented in Tables 3 and 4 . In this case, too, it was observed that in the first minutes of extraction the extraction efficiency increased with increasing microwave intensity (Figures $1 \mathrm{~d}$ and $3 \mathrm{~d}$ ). This was more evident in the case of AS extraction which started with lower yields at low intensities (Figure 3d).

Finally, interaction between extraction power (C) and ratio of water (\%) to extracted material (D) was found to be also significant (Tables 3 and 4). Figures 1e and 4c show that when the ratio of water (\%) to avocado by-product is increased, the volume of the extracted material also increases. AP shows an immediate reduction in the rate of the optimized extracts at $10.02-16.45 \%$. In the case of avocado seeds, this observation occurs when the percentage of the extraction reaches $37.65-38.73 \%$. This difference between the two by-products (which is approximately reach $22 \%$, results not shown) is near to the difference in moisture percentage of AP and AS [40].

In another study it was also shown that the microwave power had significant influence on TPC which may be attributed to the increased solubility of phenolic compounds as a 
result of increasing power which promotes cell rupture and enhances exudation of phenolic compounds into the extracting solvent [45].

\section{Conclusions}

In this research study, vacuum microwave aqueous-assisted extraction parameters of temperature, time, microwave power and water to solid ratio of avocado peel and seeds has been used successfully to optimize a microwave industrial extractor. Operational cost (estimated as extraction time) was under consideration in order to achieve optimal extraction parameters leading to high rates of extracts with high TPC and high antioxidant activity, based on measurements of the $\mathrm{IC}_{50}$ of DPPH scavenging. Under these optimal extraction conditions, we obtained high rates of AP and AS extraction with high TPC values (0.352 $\mathrm{mg} \mathrm{GAE} / \mathrm{g}$ fresh AP/min and $0.124 \mathrm{mg} \mathrm{GAE} / \mathrm{g}$ fresh AS/min) and high antioxidant capacity $(0.104 \mathrm{~L} / \mathrm{min}$ and $0.045 \mathrm{~L} / \mathrm{min})$ of extract equivalent volume at reference $\mathrm{IC}_{50}$ of $\mathrm{DPPH}^{\bullet}$ radical scavenging activity equal to $100 \mathrm{mg} / \mathrm{L}$, respectively. High accuracy of the models examined and feasible application of the optimized process on an industrial scale resulted due to the application of verified tests regarding the optimal extraction conditions based on the experiments in triplicate and the high $\mathrm{R}^{2}$ of the models. According to the results presented in this research, VMAAE achieved the conversion of avocado by-product directly, without using previous cost-effective drying pre-treatments processes, into a valuable phytochemical ingredient for food, pharmaceutical, cosmetic and other industries, thereby providing a viable solution to avocado processing companies for the valorization of their by-products.

Author Contributions: Conceptualization, P.S. and S.L.; Data curation, P.S. and K.P.; Formal analysis, P.S. and K.P.; Investigation, P.S. and S.L.; Software, P.S and K.P.; Supervision, K.P. and I.G.; Writingoriginal draft, P.S. and S.L.; Writing-review and editing, P.S., S.L., I.G. and K.P. All authors have read and agreed to the published version of the manuscript.

Funding: The Operational Programme "Human Resources Development, Education and Lifelong Learning 2014-2020" in the context of the project "Production of natural antimicrobial substances from solid wastes of natural antimicrobial substances from solid wastes of food industrial for use in foods and plant protection" (MIS 5048533) co-financed by Greece and the European Union (European Social Fund-ESF) funded this research study.

Institutional Review Board Statement: Not applicable.

Informed Consent Statement: Not applicable.

Data Availability Statement: The data presented in this study are available on request from the corresponding author.

Acknowledgments: The authors would like to thank Pella's Nature P.Co. (Greece) for providing the industrial scale microwave extractor.

Conflicts of Interest: The authors declare no conflict of interest.

\section{References}

1. California Avocado Society. Taxonomy of the Avocado. 1986. Available online: http://www.avocadosource.com/cas_yearbooks/ cas_70_1986/cas_1986_pg_135-145.pdf (accessed on 15 January 2021).

2. Bhuyan, D.J.; Alsherbiny, M.A.; Perera, S.; Low, M.; Basu, A.; Devi, O.A.; Barooah, M.S.; Li, C.G.; Papoutsis, K.; Low; et al. The Odyssey of Bioactive Compounds in Avocado (Persea americana) and their Health Benefits. Antioxidants 2019, 8, 426. [CrossRef] [PubMed]

3. Hanani, Z.A.N. Gelatin. In Encyclopedia of Food and Health; Academic Press: Cambridge, MA, USA, 2016; pp. 191-195. ISBN 978-0-12-384953-3.

4. Tremocoldi, M.A.; Rosalen, P.L.; Franchin, M.; Massarioli, A.P.; Denny, C.; Daiuto, É.R.; Paschoal, J.A.R.; Melo, P.S.; De Alencar, S.M. Exploration of avocado by-products as natural sources of bioactive compounds. PLOS ONE 2018, 13, e0192577. [CrossRef]

5. Semper, C.; Zoologisch-Zootomischen Institut. Arbeiten aus dem Zoologisch-Zootomischen Institut in Würzburg. In Arbeiten aus dem Zoologisch-Zootomischen Institut in Würzburg; Stahel'schen Buch- \& Kunsthandlung: Würzburg, Germany, 1874. 
6. Melgar, B.; Dias, M.I.; Ciric, A.; Sokovic, M.; Garcia-Castello, E.M.; Rodriguez-Lopez, A.D.; Barros, L.; Ferreira, I.C. Bioactive characterization of Persea americana Mill. by-products: A rich source of inherent antioxidants. Ind. Crop. Prod. 2018, 111, 212-218. [CrossRef]

7. Soong, Y.-Y.; Barlow, P.J. Antioxidant activity and phenolic content of selected fruit seeds. Food Chem. 2004, 88, 411-417. [CrossRef]

8. Kosińska, A.; Karamać, M.; Estrella, I.; Hernández, T.; Bartolomé, B.; Dykes, G.A. Phenolic Compound Profiles and Antioxidant Capacity of Persea americana Mill. Peels and Seeds of Two Varieties. J. Agric. Food Chem. 2012, 60, 4613-4619. [CrossRef] [PubMed]

9. López-Cobo, A.; Gómez-Caravaca, A.M.; Pasini, F.; Caboni, M.F.; Segura-Carretero, A.; Fernández-Gutiérrez, A. HPLC-DAD-ESIQTOF-MS and HPLC-FLD-MS as valuable tools for the determination of phenolic and other polar compounds in the edible part and by-products of avocado. LWT 2016, 73, 505-513. [CrossRef]

10. Chel-Guerrero, L.; Barbosa-Martín, E.; Martínez-Antonio, A.; González-Mondragón, E.; Betancur-Ancona, D. Some physicochemical and rheological properties of starch isolated from avocado seeds. Int. J. Biol. Macromol. 2016, 86, 302-308. [CrossRef]

11. Ferreyra, R.; Sellés, G.G.; Saavedra, J.; Ortiz, J.; Zúñiga, C.; Troncoso, C.; Rivera, S.A.; González-Agüero, M.; Defilippi, B. Identification of pre-harvest factors that affect fatty acid profiles of avocado fruit ( Persea americana Mill) cv. 'Hass' at harvest. South Afr. J. Bot. 2016, 104, 15-20. [CrossRef]

12. Dávila, J.A.; Rosenberg, M.; Castro, E.; Cardona, C.A. A model biorefinery for avocado (Persea americana mill.) processing. Bioresour. Technol. 2017, 243, 17-29. [CrossRef] [PubMed]

13. Araújo, R.G.; Rodriguez-Jasso, R.M.; Ruiz, H.A.; Pintado, M.M.E.; Aguilar, C.N. Avocado by-products: Nutritional and functional properties. Trends Food Sci. Technol. 2018, 80, 51-60. [CrossRef]

14. Permal, R.; Chang, W.L.; Seale, B.; Hamid, N.; Kam, R. Converting industrial organic waste from the cold-pressed avocado oil production line into a potential food preservative. Food Chem. 2020, 306, 125635. [CrossRef]

15. Rasouli, H.; Farzaei, M.H.; Khodarahmi, R. Polyphenols and their benefits: A review. Int. J. Food Prop. 2017, 20, 1-42. [CrossRef]

16. Galanakis, C.M.; Galanakis, C. Recovery of high added-value components from food wastes: Conventional, emerging technologies and commercialized applications. Trends Food Sci. Technol. 2012, 26, 68-87. [CrossRef]

17. Mandal, V.; Mohan, Y.; Hemalatha, S. Microwave assisted extraction—an innovative and promising extraction tool for medicinal plant research. Pharmacogn. Rev. 2007, 1, 7-18.

18. Segovia, F.J.; Corral-Pérez, J.J.; Almajano, M.P. Avocado seed: Modeling extraction of bioactive compounds. Ind. Crop. Prod. 2016, 85, 213-220. [CrossRef]

19. Carciochi, R.A.; D'Alessandro, L.G.; Vauchel, P.; Rodriguez, M.M.; Nolasco, S.M.; Dimitrov, K. Valorization of agrifood byproducts by extracting valuable bioactive compounds using green processes. In Ingredients Extraction by Physicochemical Methods in Food; Grumezescu, A.M., Holban, A.M., Eds.; Academic Press: Amsterdam, The Netherlands, 2017; pp. 191-228.

20. Sanchez-Aldana, D.; Aguilar, C.N.; Nevarez-Moorillon, G.V.; Esquivel, J.C.C. COMPARATIVE EXTRACTION OF PECTIC AND POLYPHENOLS FROM MEXICAN LIME POMACE AND BAGASSE. Am. J. Agric. Biol. Sci. 2013, 8, 309-322. [CrossRef]

21. Veggi, P.C.; Martinez, J.; Meireles, M.A. Fundamentals of microwave extraction. In Microwave-assisted Extraction for Bioactive Compounds: Theory and Practice; Chemat, F., Cravotto, G., Eds.; Springer: Boston, MA, USA, 2012; pp. 15-52.

22. Azmir, J.; Zaidul, I.; Rahman, M.; Sharif, K.; Mohamed, A.; Sahena, F.; Jahurul, M.; Ghafoor, K.; Norulaini, N.; Omar, A. Techniques for extraction of bioactive compounds from plant materials: A review. J. Food Eng. 2013, 117, 426-436. [CrossRef]

23. Alupului, A.; Calinescu, I.; Lavric, V. Microwave extraction of active principles from medicinal plants. UPB Sci. Bull. B. 2012, 74, 129-142.

24. Chan, C.-H.; Yusoff, R.; Ngoh, G.-C.; Kung, F.W.-L. Microwave-assisted extractions of active ingredients from plants. J. Chromatogr. A 2011, 1218, 6213-6225. [CrossRef] [PubMed]

25. Xiao, X.-H.; Wang, J.-X.; Wang, G.; Wang, J.-Y.; Li, G.-K. Evaluation of vacuum microwave-assisted extraction technique for the extraction of antioxidants from plant samples. J. Chromatogr. A 2009, 1216, 8867-8873. [CrossRef]

26. Box, G.E.P.; Wilson, K.B. On the Experimental Attainment of Optimum Conditions. J. R. Stat. Soc. Ser. B 1951, 13, 1-38. [CrossRef]

27. Li, Y.; Zhu, C.-P.; Zhai, X.-C.; Zhang, Y.; Duan, Z.; Sun, J.-R. Optimization of enzyme assisted extraction of polysaccharides from pomegranate peel by response surface methodology and their anti-oxidant potential. Chin. Herb. Med. 2018, 10, 416-423. [CrossRef]

28. Skenderidis, P.; Leontopoulos, S.; Petrotos, K.; Giavasis, I. Optimization of Vacuum Microwave-Assisted Extraction of Pomegranate Fruits Peels by the Evaluation of Extracts' Phenolic Content and Antioxidant Activity. Foods 2020, 9, 1655. [CrossRef]

29. Waterhouse, A.L. Determination of Total Phenolics. Curr. Protoc. Food Anal. Chem. 2002, 6, I1.1.1-I1.1.8. [CrossRef]

30. Brand-Williams, W.; Cuvelier, M.; Berset, C. Use of a free radical method to evaluate antioxidant activity. LWT 1995, 28, 25-30. [CrossRef]

31. Wang, Y.; Liu, Y.; Hu, Y. Optimization of polysaccharides extraction from Trametes robiniophila and its antioxidant activities. Carbohydr. Polym. 2014, 111, 324-332. [CrossRef]

32. Eskilsson, C.S.; Björklund, E. Analytical-scale microwave-assisted extraction. J. Chromatogr. A 2000, 902, 227-250. [CrossRef]

33. Calderón-Oliver, M.; Pedroza-Islas, R.; Escalona-Buendía, H.B.; Pedraza-Chaverri, J.; Ponce-Alquicira, E. Comparative study of the microencapsulation by complex coacervation of nisin in combination with an avocado antioxidant extract. Food Hydrocoll. 2017, 62, 49-57. [CrossRef] 
34. Maran, J.P.; Swathi, K.; Jeevitha, P.; Jayalakshmi, J.; Ashvini, G. Microwave-assisted extraction of pectic polysaccharide from waste mango peel. Carbohydr. Polym. 2015, 123, 67-71. [CrossRef]

35. Calderón-Oliver, M.; Escalona-Buendía, H.B.; Medina-Campos, O.N.; Pedraza-Chaverri, J.; Pedroza-Islas, R.; Ponce-Alquicira, E. Optimization of the antioxidant and antimicrobial response of the combined effect of nisin and avocado byproducts. LWT 2016, 65, 46-52. [CrossRef]

36. Khajeh, M.; Moghaddam, A.R.A.; Sanchooli, E. Application of Doehlert Design in the Optimization of Microwave-Assisted Extraction for Determination of Zinc and Copper in Cereal Samples Using FAAS. Food Anal. Methods 2009, 3, 133-137. [CrossRef]

37. Li, J.; Zu, Y.-G.; Fu, Y.-J.; Yang, Y.-C.; Li, S.-M.; Li, Z.-N.; Wink, M. Optimization of microwave-assisted extraction of triterpene saponins from defatted residue of yellow horn (Xanthoceras sorbifolia Bunge.) kernel and evaluation of its antioxidant activity. Innov. Food Sci. Emerg. Technol. 2010, 11, 637-643. [CrossRef]

38. Avhad, M.; Marchetti, J.M. Temperature and pretreatment effects on the drying of Hass avocado seeds. Biomass- Bioenergy 2015, 83, 467-473. [CrossRef]

39. Morais, D.R.; Rotta, E.M.; Sargi, S.C.; Schmidt, E.M.; Bonafe, E.G.; Eberlin, M.N.; Sawaya, A.C.H.F.; Visentainer, J.V. Antioxidant activity, phenolics and UPLC-ESI(-)-MS of extracts from different tropical fruits parts and processed peels. Food Res. Int. 2015, 77, 392-399. [CrossRef]

40. Mohammed, H.N.; Beeram, E.; Divya, B.J.; Pallavi, C.; Demir, H.; Demir, B.B.; Polley, T.; Ghosh, U.; Shiekuma, S.; Ukeyima, M.; et al. Current Research in Agricultural and Food Science Vol. 1; Mastanjević, K., Ed.; Book Publisher International: London, UK, 2020; Volume 1. [CrossRef]

41. Chan, C.H.; Yeoh, H.K.; Yusoff, R.; Ngoh, G.C. A first-principles model for plant cell rupture in microwave-assisted extraction of bioactive compounds. J. Food Eng. 2016, 188, 98-107. [CrossRef]

42. Kala, H.K.; Mehta, R.; Sen, K.K.; Tandey, R.; Mandal, V. Critical analysis of research trends and issues in microwave assisted extraction of phenolics: Have we really done enough. TrAC Trends Anal. Chem. 2016, 85, 140-152. [CrossRef]

43. Trujillo-Mayol, I.; Céspedes-Acuña, C.; Silva, F.L.; Alarcón-Enos, J. Improvement of the polyphenol extraction from avocado peel by assisted ultrasound and microwaves. J. Food Process. Eng. 2019, 42, 13197. [CrossRef]

44. Dahmoune, F.; Nayak, B.; Moussi, K.; Remini, H.; Madani, K. Optimization of microwave-assisted extraction of polyphenols from Myrtus communis L. leaves. Food Chem. 2015, 166, 585-595. [CrossRef]

45. Weremfo, A.; Adulley, F.; Adarkwah-Yiadom, M. Simultaneous optimization of microwave-assisted extraction of phenolic compounds and antioxidant activity of avocado (Persea americana Mill.) seeds using response surface methodology. J. Anal. Methods Chem. 2020, 17. [CrossRef] [PubMed] 